\title{
Chancengleichheit und Leistungsmotiv in der Bildungspolitik: Die Debatten um die Gesamtschule am Beispiel Luxemburgs
}

\author{
Andreas Hadjar ${ }^{1, *}$, Barbara Rothmüller ${ }^{1}$ \\ 1 Institute of Education and Society, Université du Luxembourg, Luxembourg \\ * E-Mail: andreas.hadjar@uni.lu
}

\section{Zusammenfassung}

Chancengleichheit und Meritokratie sind in der Bildungspolitik häufig gebrauchte Schlagworte der Legitimation von Reformen. Ein wesentliches Reformelement zur Verringerung von Bildungsungleichheiten stellt seit Beginn des 20. Jahrhunderts die Gesamtschule dar. Das Gleichheits- und Leistungsverständnis hinter dieser heftig diskutierten Reformmaßnahme ist jedoch sehr unterschiedlich und bis heute umstritten. Der Beitrag analysiert zentrale bildungspolitische Argumente für und gegen eine Gesamtschulreform am Beispiel Luxemburgs. Anhand von ExpertInneninterviews und historischen Dokumenten wird gezeigt, in welcher Weise sich unterschiedliche politische Gleichheitszugänge mit einer spezifischen Leistungskonstruktion und nationalen Elitereproduktion verbinden können.

\section{Schlüsselwörter}

Chancengleichheit, soziale Ungleichheit, Leistung, Gesamtschule, Bildungsreform

\section{Equality of opportunity and merit in educational politics: The controversies over a comprehensive school in Luxembourg}

\begin{abstract}
Equality of opportunity and meritocracy are common catchwords of the legitimation of educational reforms. In the $2 \mathrm{O}^{\text {th }}$ century, a comprehensive school reform was one major reform project that was expected to reduce educational inequalities. Yet, very different concepts of equality, merit and achievement dominated the heated debates over a comprehensive school which remain controversial until today. Drawing on the example of Luxembourg, the article analyses main political arguments for and against a comprehensive school reform. Based on expert interviews and historical documents, the authors show how different political approaches to equality are connected with specific concepts of merit as well as the social reproduction of national elites.
\end{abstract}

\section{Keywords}

Equality of opportunity, social inequality, merit, comprehensive school, educational reform

The authors have declared that no competing interests exist. 


\section{Einleitung}

Das Leistungsmotiv und das Prinzip der Chancengleichheit stehen im Zentrum öffentlicher, politischer und wissenschaftlicher Debatten um Bildungsungleichheiten. Für bildungspolitische Initiativen gegen Ungleichheiten, die eine Reduktion von Benachteiligungen verschiedener Risikogruppen im Bildungserwerb anstreben, erscheint die Gesamtschule als Lösungsansatz der Wahl, um Chancengleichheit zu gewährleisten. Entsprechend bauen ganze Bildungssysteme - etwa das finnische - auf gesamtschulartigen Schulformen auf, oder werden Gesamt- bzw. Gemeinschaftsschulen neben anderen Schulformen in Bildungssysteme integriert (z.B. in vielen deutschen Bundesländern). Allerdings hat sich die Idee eines gesamtschulartigen Bildungssystems - das gering stratifiziert ist, d.h. keine oder nur eine sehr späte Selektion von SchülerInnen auf verschiedene Bildungswege im Sekundarbereich vornimmt - nicht flächendeckend durchgesetzt. Ein Grund dafür ist in kontextspezifischen bildungspolitischen Aushandlungsprozessen zu sehen, die sich in nationalen und regionalen politischen Debatten für bzw. gegen die Gesamtschule mit unterschiedlichen Schwerpunktsetzungen seit mehreren Jahrzehnten beobachten lassen. Ein interessantes Beispiel zum Verständnis diskursiver bildungspolitischer Bruchlinien von Gesamtschulreformen liefert Luxemburg, wo 1979 zwar die Gesamtschule von der sozialdemokratischen Regierung nach heftigen Auseinandersetzungen gesetzlich verankert wurde, jedoch schließlich am konservativen Widerstand in der Praxis scheiterte. Dabei wird sichtbar, dass es bei der Reform in (zumindest) zweierlei Hinsicht auch um Fragen der Leistung ging: Die Leistung der Gesamtschulreform bemaß sich für die AkteurInnen nicht zuletzt daran, welchen Einfluss eine Veränderung des Bildungssystem auf die Leistung der SchülerInnen nehmen würde.

Der vorliegende Beitrag hat zum Ziel, den bildungspolitischen Gesamtschuldiskurs im Hinblick auf die Prinzipien der Leistung und der Chancengleichheit zu analysieren. Der Fall der (gescheiterten) luxemburgischen Gesamtschulreform dient dabei der Illustration bildungspolitischer Aushandlungsprozesse, die zwar kontextspezifisch sind, durchaus jedoch Ähnlichkeiten zu den diskursiven Reformverläufen anderer Länder aufweisen. Folgende Fragen stehen dabei im Zentrum: Was bedeuten die Prinzipien der Leistung und der Chancengleichheit im Hinblick auf Makro-Charakteristiken des Bildungssystems, insbesondere die Frage der Mehrgliedrigkeit? Wie positionieren sich unterschiedliche bildungspolitische AkteurInnen $\mathrm{zu}$ Leistung und Gleichheit? Wie finden sich diese bildungspolitischen Positionen schließlich in konkreten bildungssystemischen Reformpolitiken wieder?

Im Rahmen dieses Beitrags werden zunächst Leistungsmotiv, meritokratisches Prinzip und Ideal der Chancengleichheit innerhalb der Thematik der Bildungsun- gleichheiten verortet. In einem weiteren Schritt wird gefragt, inwieweit die Gesamtschule bzw. gesamtschulartige Bildungssysteme diesen Prinzipien entsprechen. Im zweiten Teil des Beitrags erfolgt dann eine Analyse der Debatten um eine Luxemburgische Gesamtschule, in der wir den Ungleichheits- und Leistungsdiskurs im Verlauf der Gesamtschulreform analysieren. Dabei kristallisieren sich Argumente hinsichtlich der Ziele der Gesamtschule sowie der Mittel, wie diese erreicht werden können (präferierte Systemmerkmale), heraus. Neben der Analyse von historischen Quellen greifen wir dazu auf leitfadengestützte ExpertInneninterviews zurück.

\section{Chancengleichheit und Leistung im Bildungssystem}

Bildungspolitische Debatten drehen sich explizit und implizit häufig um die Funktionen von Bildung. Die Leistung des Bildungssystems auf der Makroebene wird je nach Perspektive durch eine strenge Selektion auf Basis des Leistungsprinzips oder durch die Herstellung von (Chancen-)Gleichheit durch Kompensation von Benachteiligungen in der Schule garantiert. Im meritokratischen Prinzip (Young 1958) finden die Werte der Chancengleichheit und der Leistung zwar eine Verknüpfung (vgl. z.B. das Konzept von Rawls I975 [197I]), es zeigt sich aber dennoch ein Widerspruch hinsichtlich der Problematiken Gleichheit versus Ungleichheit sowie Exklusion versus Inklusion: Während mit Chancengleichheit gefordert wird, „persönlich nicht $\mathrm{zu}$ verantwortende Unterschiede zwischen den Menschen" (Meulemann 1992, IO2) wie soziale Herkunft, Geschlecht oder Ethnie nicht zur Grundlage von ungleichen Verteilungen von Gütern und Positionen zu machen, wird mit dem Leistungsprinzip wieder ein ungleichheitslegitimierender Mechanismus eingeführt. Chancengleichheit begünstigt zwar die Inklusion vorher exkludierter gesellschaftlicher Gruppen - etwa ins Bildungssystem -, das Leistungsprinzip legitimiert aber neue Ungleichheiten (etwa in der Zuweisung auf bestimmte Schullaufbahnen und spätere Positionen). Leistungsbezogene Unterscheidungen können entsprechend wiederum exkludierende Mechanismen beinhalten, da sie der Legitimation von Auswahlprozessen bzw. -entscheidungen dienen können. Das (meritokratische) Leistungsprinzip trägt somit eine Ungleichheitskomponente in sich.

Aus Perspektive einer inklusiven Bildungstheorie und - politik wird des Weiteren kritisiert, dass sich die Definition dessen, was als Leistung gilt, an den Fähigkeiten nur eines Teils der Bevölkerung orientiere (vgl. z.B. Wocken 1988). Entsprechend wird das Leis- 
tungsprinzip zum Exklusionskriterium für alle, welche den gesellschaftlich oder in bestimmten Institutionen anerkannten Fähigkeitskatalog nicht erfüllen.

Der eigentliche Gegenpol des Prinzips der Chancengleichheit bzw. des Leistungsprinzips ist der Egalitarismus mit seiner Forderung nach einer gleichen Verteilung von Gütern und Positionen - zu denen auch Bildungszertifikate gehören. So enthält beispielsweise das genuin egalitäre Prinzip des, luck egalitarianism' (Dworkin 2000; vgl. Swift 2004) die Forderung, dass Gesellschaften die Bedürfnisse aller Menschen bestmöglichst erfüllen sollen, unabhängig davon, welche Fähigkeiten und Talente diese haben (oder: zu welchen Leistungen sie fähig sind). Für das Bildungssystem würde das Gleichheitsprinzip bedeuten, dass Schule nicht auf Selektion - unter welchem Kriterium auch immer - sondern daran ausgerichtet ist, dass alle Schülerinnen und Schüler gleiche (erfolgreiche) Ergebnisse erzielen.

Die Sichtweise der Chancengleichheit und die genuin egalitäre Sichtweise unterscheiden sich auch in der Frage, zu welchem Zeitpunkt, Gleichheit' u.a. durch politische Maßnahmen hergestellt werden soll. Das „liberale Ideal" der Meritokratie bezieht sich aus Sicht von Miller (2002) auf einen Moment gleicher Startchancen statt auf kontinuierliche Chancengleichheit:

A person's opportunities have to be judged at some suitably chosen starting point, since each decision that is made to avail oneself of an opportunity, or not to do so, is likely to affect the opportunity set at a later point. For example, a person who decides to leave school at sixteen cannot later complain that she was denied the opportunity to go to university, if by staying on at school she could have achieved that goal (Miller 2002, 47).

Die Reduktion von Ungleichheiten kann aber aus der Perspektive von Chambers (2009) nur durch die Gewährleistung von Chancengleichheit über den gesamten Lebenslauf hinweg gelingen. Die fortwährende Kompensation ungleicher Bedingungen entspricht zwar dem egalitären Prinzip, steht aber durch den hohen Ressourceneinsatz Effizienzprinzipien entgegen. So spricht Chambers $(2009,396)$ von einem „trade-off [...] between justice, as secured by equality of opportunity, and meritocracy or efficiency “. ${ }^{1}$ Diese unterschiedlichen normativen Perspektiven auf Bildungsgleichheit stehen dabei in enger Verbindung mit macht- und parteipolitischen Positionierungen.

I Im Kontext des Diversity Management wird demgegenüber betont, dass Leistung auf der Basis von Diversität zu steigern sei, wirtschaftlicher Mehrwert und soziale Gerechtigkeit sich also nicht ausschließen müssen (vgl. z.B. Koall/Bruchhaben/Höher 2007).

\section{Die Gesamtschule als Bildungssystemmerkmal}

Der Ausgestaltung des Bildungssystems im Rahmen von Bildungspolitiken wird bereits seit langem eine Wirkung auf das Ausmaß von Bildungsungleichheiten, d.h. wie stark das Leistungsprinzip umgesetzt werden kann oder wie persistent sich der Einfluss askriptiver Merkmale wie der sozialen Herkunft auf den Bildungserwerb hält, zugeschrieben (Allmendinger 1989; Müller/Karle 1993). Allerdings gibt es erst in den letzten Jahren empirische Befunde, die einen solchen Einfluss identifizieren (Crul/Schnell/Herzog-Punzenberger/Wilmes/Slootman/Aparicio-Gomez 2012; Bruneforth/Weber/Bacher 2012; Hadjar/Berger 20II; Pfeffer 2008; OECD 2005). Neben weiteren Faktoren (etwa der beruflichen Spezifizität; der Frage, wer die Schullaufbahnentscheidung für die Kinder trifft oder der Standardisierung von Schulausstattung und LehrerInnenausbildung; vgl. Hadjar/ Gross 2016) richtet die bildungssoziologische Forschung vor allem den Blick auf das Merkmal der Stratifizierung. Ein hoher Stratifizierungsgrad liegt dann vor, wenn es vor allem im Sekundarbereich verschiedene parallele Bildungswege gibt (Mehrgliedrigkeit), die SchülerInnen relativ früh auf diese verschiedenen Züge aufgeteilt werden (frühe Selektion) und zwischen den verschiedenen Bildungswegen kaum gewechselt werden kann (geringe Permeabilität). Den geringsten Stratifizierungsgrad weisen gesamtschulartige Systeme auf (Müller et al. 1997), in denen SchülerInnen die längste Zeit im gleichen Schultyp bzw. auf dem gleichen Schulniveau miteinander lernen.

Der Großteil der aktuellen Forschung teilt die Annahme, dass Bildungsungleichheiten in stratifizierten Systemen stärker ausgeprägt sind als in den wenig stratifizierten Systemen, die auf einem Gesamtschul-Modell aufbauen (van de Werfhorst/Mijs 20Io). Warum ist ein solcher Einfluss zu vermuten? Zunächst einmal zeigen sich Unterschiede nach askriptiven Merkmalen wie sozialer Herkunft oder Geschlecht vor allem an Bildungsübergängen, die mit einer Selektion verbunden sind und wie Weichen für den weiteren Bildungs- und Lebensweg funktionieren. Im klassischen stratifizierten, deutschen oder österreichischen Modell etwa wurden Kinder in der Regel nach dem Io. Lebensjahr aufgeteilt, wobei Arbeiterkinder geringere Chancen als Akademikerkinder hatten, Bildungswege zu beschreiten, die in eine Hochschulzugangsberechtigung oder sogar einen Hochschulabschluss mündeten. In gesamtschulartigen Systemen gibt es weniger und viel später positionierte Selektionspunkte. Ein früher Selektionszeitpunkt fördert auch deshalb Bildungsungleichheiten, weil in jungen Jahren der Einfluss des Elternhauses auf den Bildungserwerb und die Bildungsentscheidungen (sekundäre Effekte; Boudon 1974) noch sehr stark ist und erst später abnimmt (Müller et al. 1997; von Below 2009). Bei früher 
Selektion ist zudem die Prognose von Eltern und LehrerInnen hinsichtlich der kognitiven Entwicklung des Kindes ungenauer, was durch die Orientierung an gruppenspezifischen Stereotypen, etwa der Annahme, dass Arbeiterkinder für die Berufsbildung geeigneter sind als für eine akademische Ausbildung, kompensiert wird.

Ein weiteres Argument, das sich auf Bildungsentscheidungen bezieht, fokussiert auf das strategische Wissen der Eltern über das Bildungssystem. Während im gesamtschulartigen System dieses Wissen aufgrund fehlender oder spät positionierter "Weichen“ weniger ins Gewicht fällt, kommen in stratifizierten Bildungssystemen (etwa klassenspezifische) Wissensressourcen zum Tragen (Pfeffer 2008). Kinder begeben sich nach dieser Argumentation häufig auf den Bildungsweg ihrer Eltern, weil diese sie besser durch das "Labyrinth“ der selbst durchlaufenen Bildungsinstitutionen führen können. Fehlende Erfahrungen der Familie mit dem Hochschulsystem würden Arbeiterkinder entsprechend abschrecken, einen solchen Weg zu beschreiten.

Ein anderer Mechanismus hinter dem Zusammenhang zwischen Stratifizierungsgrad und Bildungsungleichheiten ergibt sich aus einer sozialökologischen Perspektive der Schule als Sozialisations- und Lernumfeld bzw. differentiellem Entwicklungsmilieu (Baumert et al. 2006). Zum einen bedeutet die Gesamtschule eine höhere Standardisierung hinsichtlich der Ausstattung, aber auch eine höhere Heterogenität der Schülerschaft in der Schule und den einzelnen Klassen. Nach den Erkenntnissen des Coleman-Reports (Coleman et al. 1966) können Unterschiede im Bildungserwerb, etwa Nachteile von Arbeiter- und Migrantenkindern, in gemischten und inklusiven Gesamtschulformen ausgeglichen werden, solange die Kinder mit benachteiligten Hintergründen nicht die Mehrheit der Klasse bilden. Ein bedeutsamer Mechanismus dieser Befunde ist sicher, dass in niedrigen Schulzügen wie der deutschen Hauptschule oder in Schweizer Realklassen eine homogene SchülerInnenpopulation aus benachteiligten Hintergründen (Überrepräsentation von Arbeiterkindern, Jungen, Kindern mit Migrationshintergrund hinsichtlich ihrer gesamtgesellschaftlichen Prozentanteile) zu finden ist. Die Risikogruppen, vor allem die Arbeiterkinder und Jungen, bleiben somit unter sich und bestärken sich gegenseitig in geringer Motivation und können sich auch weniger als andere gegenseitig bei Lernaktivitäten unterstützen (Hadjar/Berger 2010). Somit werden Defizite im Sinne primärer Effekte nach Boudon (1974), d.h. auf Nachteilen in der Ressourcenausstattung basierende geringere Schulleistungen, nicht ausgeglichen. Zudem besteht die Möglichkeit, dass LehrerInnen in den entsprechenden Schulen oder Klassen ein niedrigeres Anspruchsniveau anlegen. D.h. familiär begründete Unterschiede im Bildungserwerb können in mehrgliedrigen Systemen weniger erfolgreich reduziert werden, sondern werden stattdessen zementiert (Baumert et al. 2006; Schubert/ Becker 20IO). Kritisch lässt sich anmerken, dass dabei die Frage, was als Leistung und Fähigkeit im schulischen Kontext anerkannt wird, kaum adressiert wird. Anstelle der Defizite benachteiligter Kinder könnte auch auf ihre Ressourcen fokussiert werden, zum Beispiel eine etwaige Mehrsprachigkeit, die jedoch bislang in vielen Bildungssystemen (noch) wenig geschätzt wird. An dieser Stelle setzt die oben erwähnte Frage der Leistungsdefinition ein, die diskursiv wiederum selbst eine Rolle in der Er- bzw. Entmutigung bestimmter Bildungsgruppen bei der Entwicklung ihrer je spezifischen Fähigkeiten, Motivationen und Bildungsentscheidungen spielen kann (siehe für ein Beispiel Rothmüller 20I4).

Schließlich ist auch auf die unterschiedliche Ressourcenausstattung der verschiedenen Schultypen in mehrgliedrigen Bildungssystemen hinzuweisen. Entsprechend einer politisch durchgesetzten gesellschaftlichen Wertschätzung für die einzelnen Schultypen werden Mittel und Personen (Lehrpersonen, Unterstützungspersonal) allokiert, traditionell waren dabei die "niedrigeren“ Schultypen hinsichtlich ihres eigentlich höheren Unterstützungsbedarfs unterprivilegiert (bis im letzten Jahrzehnt auch das öffentliche Augenmerk auf "Problemschulen“" gerichtet wurde). Die Ressourcenausstattung ist in Österreich und Deutschland eher heterogen und hat eine wichtige Bedeutung für die SchülerInnenleistungen (OECD 2OII).

Wie bereits erwähnt ist der Forschungsstand zur Frage, ob gesamtschulartige Systeme durch geringere Bildungsungleichheiten gekennzeichnet sind, nicht sehr breit. Allerdings haben sowohl Analysen der PISADaten (Stelzer-Orthofer/Bacher 2008; OECD 2005) als auch des europäischen Vergleichsprojektes zur Integration der Zweiten Generation TIES (Crul et al. 2012) auf den Einfluss des Stratifizierungsgrads des Bildungssystems hingewiesen (für internationale Befunde siehe u.a. Hadjar/Gross 2016). Während die auf Mehrgliedrigkeit setzenden Bildungspolitiken in den meisten deutschen Bundesländern, den deutschsprachigen Schweizer Kantonen und in Österreich offenbar mit starken Bildungsungleichheiten verbunden sind, d.h. hier die Unterschiede im Bildungserwerb zwischen Arbeiter- und Akademikerkindern besonders groß sind, zeigen die gesamtschulartigen nordischen Länder die geringsten Bildungsungleichheiten (Müller et al. 1997). Mehrgliedrigkeit scheint mit mehr Ungleichheit verbunden zu sein, d.h. der Stratifizierungsgrad des Bildungssystems ist negativ mit Chancengleichheit verknüpft, wie auch die Meta-Analyse der OECD (2005), von Stelzer-Orthofer und Bacher (2008), von Werfhorst und Mijs (2010) oder Befunde von Pfeffer (2008) bzw. Hadjar und Gross (2016) zeigen. Unberücksichtigt bleibt in diesen Systemvergleichen oft, weshalb historisch in bestimmten Ländern stratifizierte und in anderen Ländern Gesamtschulsys- 
teme umgesetzt wurden. Die allgemein für Reformpolitiken zentralen Parteienwettbewerbsdynamiken und Regierungszyklen einerseits bzw. Berufsstandesinteressen und klientelistische Politik andererseits haben auch Erklärungskraft für den Verlauf bildungspolitischer Reformprozesse (Benz 2009, IIgf.), wie das Fallbeispiel Luxemburg zeigt.

\section{Die Gesamtschule als Rezept gegen Ungleichheiten?}

Gesamtschulreformen haben eine lange Tradition und wurden vor allem in der zweiten Hälfte des 20. Jahrhunderts umgesetzt. Die Legitimationsmuster, die die Debatten um eine Gesamtschule prägten, und der Erfolg der Reforminitiativen sind in den europäischen Ländern unterschiedlich ausgeprägt, so dass ein näheres Verständnis dieser bildungspolitischen Aushandlungsprozesse eine kontextsensible Analyse erfordert.

Bevor das Gleichheits- und Leistungsverständnis in der bildungspolitischen Debatte um eine Gesamtschule am Beispiel Luxemburgs im Detail rekonstruiert wird, soll deshalb kurz auf den historischen Kontext der Gesamtschulreformen und einige Spezifika der luxemburger Bildungsstruktur eingegangen werden. Die nachfolgende Diskursanalyse der Debatte rekonstruiert Diskursstrategien verschiedener ReformakteurInnen und analysiert einen Datenkorpus, in den sowohl historische Quellen als auch Interviewmaterial Eingang fanden. (Landwehr 20IO; Nullmeier 200I). Neben medialen, bildungspolitischen und pädagogischen Quellen (u.a. Tageszeitungen, Parlamentsdebatten und LehrerInnenzeitschriften) wurden I5 leitfadengestützte ExpertInneninterviews (Bogner/Littig/Menz 2005) mit verschiedenen bildungspolitischen AkteurInnen einbezogen, die im Zeitraum von März bis Juni 2015 in Luxemburg geführt wurden. ${ }^{2}$ Die Befragten waren u.a. im Luxemburger Bildungsministerium, in Parteien, Gewerkschaften oder in LehrerInnenverbänden sowie in Programmkommissionen tätig. Die Auswertung der Daten für den vorliegenden Beitrag konzentriert sich auf die diskursiven Aushandlungsprozesse, die dem Scheitern der Reform vorangingen. Zum besseren Verständnis des Reformverlaufs werden Rechtfertigungsweisen und Kritik der geplanten Reform herausgearbeitet und zu bildungspolitischen Interessen der ReformakteurInnen in Beziehung gesetzt.

2 Der Großteil der Befragten, die in den I970er und I980er-Jahren in die Bildungsreform involviert waren, war zum Zeitpunkt der Interviews bereits in Pension. Die zur anonymisierten Kennzeichnung der InterviewpartnerInnen verwendeten Positionen beziehen sich daher auf frühere Berufspositionen und wichtige politische Funktionen. Sechs der 15 Interviews wurden dabei gemeinsam mit Ragnhild Barbu und Matias Gardin geführt..

\subsection{Europäische Gesamtschul-Reforminitiativen im} 20. Jahrhundert

Die Idee der Gesamtschule hat ihre Wurzeln in sozialdemokratischem Denken und Politiken und rückte bereits Anfang bis Mitte des 20. Jahrhunderts in einigen europäischen Ländern ins Zentrum der bildungspolitischen Debatte. Die Gesamtschulidee wurde 1906 am Mannheimer Parteitag der SPD in Deutschland von Heinrich Schulz und Clara Zetkin in ihrem Beitrag zu „Volkserziehung und Sozialdemokratie" als radikale sozialdemokratische Version einer "Einheitsschule“ konzipiert (Keim 2004). Weniger radikal wurde in Österreich von dem sozialdemokratischen Unterrichtsminister Otto Glöckel Anfang der I92oer Jahre das (gesamtschulartige) Modell einer Allgemeinen Mittelschule vorgeschlagen, um soziale Ungleichheit und Bildungsprivilegien durch gemeinsamen Unterricht in zumindest den $\mathrm{Ne}-$ benfächern zu reduzieren (Bucher 20I3, 78f.). Die skandinavischen Länder begannen schließlich ab 1950 ihre differenzierten Bildungssysteme in Gesamtschulsysteme umzubauen, mit dem Ziel, soziale und regionale Bildungsungleichheiten zu reduzieren und allen SchülerInnen gleiche Bildungschancen zu bieten. Allgemeines Vorbild für die Gesamtschulreformen in Europa wurde Schweden, wo 1962 eine 9-jährige Grundschule eingeführt wurde, und zwar in Form einer Gesamtschule ohne Fachleistungsdifferenzierung und mit Ganztagesbetreuung (Ratzki 2003). Die ersten Schulversuche wurden in Schweden bereits 1950 gestartet und Kompromisse mit den konservativen GesamtschulkritikerInnen gesucht, was dazu beitrug, dass schließlich sogar die Konservative Partei der Einführung zustimmte (ebd., I28). Als sogenannte "rollende Reform“ folgte sie dem „Konzept einer egalitär-demokratischen Gesellschaft, das die Sozialdemokratie vertrat" (ebd.). In Frankreich wurde 1975 das Collège als französische Variante einer gemeinsamen Schule der II- bis I4-Jährigen eingeführt, nachdem konkrete Initiativen zur Umgestaltung des Systems bereits seit 1963 bestanden hatten (Werry 2003).

Zentraler Anstoß einer „Hochzeit der Gesamtschulen" in den I970er-Jahren in Deutschland, Österreich und Luxemburg ging von den Empfehlungen des Deutschen Bildungsrates (1969) zur „Einrichtung von Schulversuchen mit Gesamtschulen" aus (Bucher 20I3, 79; Keim 2004). Die Gesamtschule sollte Ungleichheiten verringern und sich nicht in erster Linie an einem „Verwertbarkeitsprinzip von Bildung“, sondern am "ganze(n) Spektrum menschlicher Leistung" orientieren, wie ein Gesamtschulproponent 1972 formulierte (zit. in Herrlitz 2013, I2). Dieses Spektrum umfasse u.a. Solidarität, „soziale Sensibilität" sowie „Kritikfähigkeit“, weshalb sozialwissenschaftliche Fächer verstärkt in den Lehrplan aufgenommen werden sollten (ebd.). In Deutschland gab es zwar in der DDR nach dem 2. Weltkrieg eine achtjährige 
und später zehnjährige Einheitsschule für alle Kinder, in der BRD jedoch konnte trotz Interventionen der USamerikanischen BesatzerInnen keine über die Grundschule hinausgehende gemeinsame Schule durchgesetzt werden (Wenzler 2003). Die schließlich in den I970er Jahren in der BRD eingeführten Gesamtschulversuche wurden Anfang der I980er Jahre evaluiert, und 1982 als Regelschulen bundesweit rechtlich legitimiert - neben den bestehenden anderen Schulformen.

Im Zuge der Reformprozesse kam es in fast allen Ländern zu „weltanschaulich bestimmten Grabenkämpfen“ (Bönsch 2006, 30) zwischen sozialdemokratischen und konservativen ReformakteurInnen, wie am Beispiel von Luxemburg im Folgenden historisch gezeigt werden soll.

\subsection{Das Bildungssystem Luxemburgs}

Das Luxemburgische Bildungssystem, das vielen Wandlungen unterworfen war, ist in Luxemburg traditionell stark stratifiziert, d.h. es existieren mehrere distinkte Schulwege im Sekundarschulbereich, die aufgrund des Scheiterns der Gesamtschulinitiative in Luxemburg auch stark differenziert geblieben sind. Die Schulpflicht beginnt mit dem 4. Lebensjahr mit dem Eintritt in den Vorschul-Zyklus der Grundschule und endet mit dem I5. bzw. I6. Lebensjahr. SchülerInnen werden nach dem achten Jahr in Luxemburgischen Bildungseinrichtungen (cycle 4.2), d.h. im Alter von zwölf Jahren, auf verschiedene Sekundarschulzweige aufgeteilt, die sich vereinfacht unterscheiden lassen in einen kurzen berufsbezogenen Schulweg mit dem Ausscheiden aus dem Bildungssystem nach elf Jahren Schule (gerechnet ab der Vorschule) (préparatoire), verschiedene technische Sekundarschulzweige mit dem Ziel von Berufsabschlüssen oder spezifischen (Fach-)Hochschulreifen (l'enseignement secondaire technique) und dem klassischen allgemeinbildenden Weg zur Hochschulzugangsberechtigung (l'enseignement secondaire) (MENEJ/Université du Luxembourg 2015). Ein besonderes Charakteristikum ist auch die Mehrsprachigkeit, die sich aus den drei Amtssprachen des Großherzogtums ergibt: Während in der Vorschule Lëtzebuergesch (eine moselfränkische Sprachvarietät des Westmitteldeutschen) gesprochen wird, findet die Alphabetisierung in der Grundschule in Deutsch statt. Nach der Einführung des Französischen werden in einigen Sekundarschulzügen verschiedene Fächer in Französisch unterrichtet. Bildungsungleichheiten sind stark ausgeprägt, sowohl was die Aufteilung auf die verschiedenen Sekundarschulformen als auch was die Kompetenzen und Schulnoten angeht. $\mathrm{Zu}$ den entsprechend benachteiligten Gruppen gehören sozioökonomisch benachteiligte Gruppen (Arbeiterkinder), Jungen und SchülerInnen mit bestimmten Migrationshintergründen (z.B. PortugiesInnen) (MENFP/EMACS 20IO; Hadjar et al. 2015).

\subsection{Die (gescheiterte) Reform einer Gesamtschule in Luxemburg}

Es waren vor allem die starken sozioökonomischen Ungleichheiten im Luxemburger Bildungssystem, die ab 1970 ein heftig diskutiertes Thema wurden, als erste Studien über die gering ausgeprägte Chancengleichheit in der Luxemburger Gesellschaft veröffentlicht wurden. $\mathrm{Ab}$ I974 übernahm in Luxemburg eine Koalition der sozialdemokratischen Luxemburger Sozialistischen Arbeiterpartei (LSAP) und der liberalen Demokratischen Partei (DP) die Regierung. Sie strebte von Beginn an eine bildungspolitische Wende zu mehr Chancengleichheit durch eine Gesamtschulreform an. Auf Regierungsinitiative wurde eine Kommission gebildet, die mit der Ausarbeitung von konkreten Umsetzungsvorschlägen beauftragt wurde. Im Jahr 1978 wurde in insgesamt sechs Parlamentssitzungen über den Entwurf des Gesamtschulgesetzes diskutiert, bevor es 1979 verabschiedet wurde - nur wenige Wochen vor den Neuwahlen, welche die konservative Christlich Soziale Volkspartei (CSV) gewann.

Im öffentlichen und politischen Diskurs war die Gesamtschule von Anfang an mit Gleichheitsfragen eng verbunden. Sie war geplant als „eine Art Dreijahresübergang mit Orientierung“, bei der „die Auslese nicht auf [Basis von] soziokulturellen Kriterien geschehen (sollte)", wie ein/e InterviewpartnerIn ausführt (LehrerIn, leitende Funktion in sozialdemokratischer Partei, Z. 32-33). Chancengleichheit war erstes Ziel des Gesamtschulgesetzes und fungierte in der Reformdebatte als "Schlachtruf" (Bulletin IR I973, I9), bei dem vermeintlich zwischen allen AkteurInnen Einigkeit herrschte. Eine Rekonstruktion des Gleichheitsbegriffs im Diskurs der I970er Jahre zeigt aber, dass sozialdemokratische, kritisch-linke und konservative BildungsakteurInnen ganz Unterschiedliches unter Gleichheit verstanden und auch unterschiedliche Probleme an diesen Begriff knüpften.

\section{Bildungsgleichheit und Demokratisierung}

Für die GesamtschulbefürworterInnen stellte Chancengleichheit ein mit demokratischer BürgerInnenschaft verknüpftes Ideal dar. Bildungsgleichheit und Demokratisierung waren zwar schon seit den I960er-Jahren bildungspolitisch angestrebte Ziele, ihre Realisierung wurde aber in den I970ern verstärkt eingefordert, als eine Studie zum Bildungserwerb (MAGRIP) erschien, die starke soziale Ungleichheiten beim Bildungszugang in Luxemburg nachwies. Die Gesamtschule sollte Demokratisierung und eine Verringerung der Chancenungleichheit leisten. Demokratisierung wurde dabei verstanden als das Anheben des allgemeinen kulturellen Niveaus für die größtmögliche Anzahl an Personen, nicht zuletzt um den Herausforderungen der Wirt- 
schaftskrise durch höhere Qualifikation der Bevölkerung zu begegnen (Courrier de l'éducation nationale I977, IO2f.). Chancengleichheit sollte die Gesamtschule im Sinne eines gleichen Zugangs zu Bildung verwirklichen - und nicht als eine Gleichheit der Ergebnisse, wie eine Regierungsaussendung klarstellte (ebd., A96). Die Kritik am Leistungsprinzip spielte für die Legitimation einer Gesamtschulreform eine wesentliche Rolle, wie ein damals aktives Mitglied des Vereins der SekundarschullehrerInnen ausführt:

Zuerst würde ich sagen, das klang am Anfang sehr stark in den Debatten, das sogenannte klassische Schulsystem stand im Mittelpunkt und das wurde dann sehr scharf kritisiert. Als Beispiel das Leistungsprinzip im klassischen Unterricht, das ist negativ zu beurteilen. Das ist eigentlich die erste Überlegung, es ist zu rigid, Leistung Leistung Leistung, und davon profitieren die besten Schüler, aber die anderen bleiben auf der Strecke. Und es gab immer wieder eine Zahl: 40 Prozent der Schüler verlassen die Schule ohne Schulabschluss. Das Argument wird immer wieder von den Vertretern, von den Befürwortern des tronc commun, der Gesamtschule, vorgebracht. Und es gibt so viele Misserfolge in dem klassischen Unterricht, das gehört natürlich dazu, und besonders die Schüler aus sozial benachteiligten Schichten, die sind in diesem rigiden System benachteiligt, das war das sehr wichtige Argument. (LehrerIn, Verein der SekundarschullehrerInnen, Z. 29-39)

Kritik an der Gesamtschule wurde aus mehreren Richtungen formuliert: Einerseits aus dem linken politischen Lager, ${ }^{3}$ zentrales Argument war hier die problematische Isolierung von Bildungsgleichheit von gesellschaftlicher Gleichheit. Die Regierung arbeite mit einem „stark reduzierten Begriff von Chancengleichheit", kritisierte ein linker LehrerInnenverein (Bulletin CLEC I979, 9). Chancengleichheit im Bildungsbereich habe vielmehr eine "gesellschaftspolitische Dimension“, nämlich soziale Mobilität zu erhöhen (ebd.). Bildungsreformen ohne Gesellschaftsreformen könnten alleine, so die Kritik, keine soziale Gleichheit sicherstellen, weshalb sie als Frage der "Erziehung im Kapitalismus“ zu diskutieren seien (d'Letzeburger Land 1977a). Im Zuge der Debatte wurden verstärkt auch die bildungssoziologischen Arbeiten von Pierre Bourdieu (z.B. Bourdieu/Passeron I97I) und Raymond Boudon (1974) rezipiert und ihre Kritik an

3 Die Kritik kam dabei u.a. von Personen rund um den Cercle de liaison des enseignants critiques (CLEC), einem "Kreis der kritischen Lehrer" aus dem Spektrum SozialdemokratInnen, KommunistInnen, LinksLiberale, MaoistInnen und TrotzkistInnen. Jene aus der Gruppe, die nicht mit Regierungswechsel ins Ministerium berufen wurden, äußerten sich in der Folge öffentlich kritisch gegenüber den Reformplänen der Regierung und es kam schließlich „zu einer Spaltung zwischen denen, die das Gesamtschulprojekt der Regierung mittrugen, und denen, die das völlig ungenügend fanden", wie ein/e befragte LinkspolitikerIn berichtet (LehrerIn, LinkspolitikerIn, Z. 312-314). mangelnder Chancengleichheit in Bildungsinstitutionen auf Luxemburg bezogen (d'Letzeburger Land 1977a, 1977b). Auch die zunehmende Legitimation der Gesamtschule durch volkswirtschaftliche Qualifikationsbegründungen statt durch Gerechtigkeitserwägungen wurde kritisiert, sowie das Weiterbestehen autoritärer Entscheidungsstrukturen an Schulen, die mit einer Demokratisierung unvereinbar wären (Bulletin CLEC 1979, I5). Es gehe der Reform in erster Linie um „das Erlernen einer flotten Arbeitsmoral und die Verinnerlichung des Leistungsprinzips" (ebd., I2).

Kritik an der Gesamtschule wurde andererseits aus dem konservativen politischen Lager formuliert, insbesondere von der Christlich-sozialen Partei (CSV) und rund um den Verband der SekundarschullehrerInnen (APESS). Die konservative Gleichheitskritik richtete sich gegen die bildungspolitische "Gleichschaltung" und "Indoktrinierung“ der Kinder; gegen eine "gleichmacherische Einordnung des Individuums ins Kollektiv“ (Parlamentssitzung 13.03.1979, 3839) in der sogenannten sozialistischen "Eintopfschule“ (Parlamentssitzung 15.03.1979, 3932). Das am häufigsten genannte Argument von konservativen GesamtschulgegnerInnen bezog sich auf die negativen Auswirkungen dieser "Gleichschaltung" auf die schulische Leistung, die nationalspezifisch vor allem im Bereich der Sprachenkenntnisse befürchtet wurden (vgl. z.B. Bulletin APESS 1978, IO; école et vie 1976, II). „Bedenken“, so ein/e InterviewpartnerIn, richteten sich vor allem "darauf, dass im Programm die Sprachen und die Mathematik reduziert wurden zugunsten von Sozialwissenschaften“. Die SekundarlehrerInnen warnten gemeinsam mit ElternvertreterInnen vor einem Niveauverlust und fürchteten „eine Gleichschaltung in der Mittelmäßigkeit, ein Massaker der Sprachen, eine Abschaffung des Leistungsprinzips, das es nun mal im Leben gebe“" (Bulletin APESS I978, IO). Dass diese Angst vor einem "nivellement vers le bas" das zentrale Argument gegen die Gesamtschule war, wurde in den ExpertInnengesprächen deutlich zum Ausdruck gebracht:

Und zum Beispiel als Argument wurde besonders von der APESS (Verein der SekundarschullehrerInnen, Anm. BR) aus darauf hingewiesen, drei Jahre lang gemeinsam erziehen, da besteht die Gefahr, dass es zu einer Gleichmacherei kommt. Die stärkeren Schüler fühlen sich irgendwie, man könnte fast sagen, frustriert, und den Schwächeren, denen geht's auch nicht so ganz gut. D.h., also weil es keine Differenzierung gab, im ersten Jahr gibt's keine Differenzierung, im zweiten... erst im dritten Jahr werden die Schüler auch eingeteilt in schwach, mittelmäßig und stark. Und das ist also das Wort der Gleichmacherei, war natürlich damals besonders beliebt, ne, und dass auch was in Luxemburg immer wieder hervorgehoben wurde: Nivellement vers le bas, also das Niveau geht runter. (LehrerIn, Verein der SekundarschullehrerInnen, Z. 6I-73) 
Ein/e befragte/r ExpertIn fasst die von zwei Seiten kommende Kritik an der Gesamtschulinitiative zusammen: „(D)en Konservativen ging die Reform zu weit, also für die CSV und die konservativen Lehrer ging es zu weit. Und, den Linken ging es dann nicht weit genug." (LehrerIn, LinkspolitikerIn, Z. 315-7)

\section{Leistung im multilingualen Bildungskontext:}

Differenzierung und die Angst vor einem Niveauverlust Bei den genannten bildungspolitischen Perspektiven auf Gleichheit muss berücksichtigt werden, dass sie mit spezifischen Positionierungen in der Frage der Leistungsund Talenteförderung einhergehen. Während die Regierung Intelligenz und Fähigkeiten nicht als statische und unveränderbare Eigenschaften verstanden wissen wollte (vgl. Courrier de l'éducation nationale 1977, A96), schien für konservative ReformkritikerInnen eine Entwicklung von Begabungen nur begrenzt möglich, und auch nicht wünschenswert. Vielmehr sollte man die Kinder dabei unterstützen, das zu werden, was sie bereits sind - und das wäre eben Unterschiedliches (Bulletin spécial APESS 1980, 68). Während die guten Kinder sich in einer gemeinsamen Schule langweilen würden, seien die schlechten überfordert. Deshalb müsse eine frühzeitige Trennung der Kinder je nach Leistung erfolgen.

Verschiedene Formen interner Differenzierung waren jedoch immer schon wesentliches Merkmal von Gesamtschulmodellen. Die Regierung wehrte sich entsprechend in einer Aussendung dagegen, dass eine Uniformisierung der Kinder Ziel der Gesamtschule wäre. Im Gegenteil sehe das luxemburgische Gesamtschulkonzept eine interne Differenzierung vor, und zwar abhängig von Schulfach und Stufe eine Differenzierung der Kinder nach Leistung, Wahl und/oder Interessen (Courrier de l'éducation nationale 1977, A78, A88f.). Die Gesamtschule wurde mit dem Slogan „Integrieren, um besser zu differenzieren " ${ }^{4}$ beworben und dem Argument eines Niveauverlustes mit Hinweis auf eine allgemein bessere Qualifizierung der Bevölkerung in einer Gesamtschule widersprochen (ebd., A55, AIO2f.).

Seitens des linken LehrerInnenvereins wurde der Regierung in der Folge vorgeworfen, sich im Gesetzesentwurf mit der geplanten Leistungsdifferenzierung und der Betonung von Begabtenförderung dem konservativen Druck gebeugt zu haben und keine wirklich ,egalitäre Reform“ mehr zu verfolgen (vgl. Bulletin CLEC I979, 2), worunter idealerweise eine Einheitsschule bis zum Alter von I8 Jahren verstanden wurde. Die Vorschläge des Unterrichtsministeriums seien daher als „Verrat an einem fortschrittlichen Konzept" zu werten (ebd., I5).

Die zentralen diskursiven Bruchlinien der Gesamtschuldebatte verliefen somit zwischen einem Verständnis von Bildungsgleichheit als Startchancengleichheit,

4 Im Orig. franz.: „Intégrer pour mieux différencier“.
Ergebnisgleichheit, oder aber "Gleichmacherei“. Während sowohl die VerfechterInnen des liberalen Begriffs von Chancengleichheit als gleiche Startchancen, als auch diejenigen, die auf das sozialistische Gerechtigkeitsideal gleicher Ergebnisse fokussieren, letztlich eine Verringerung von sozialen Ungleichheiten anstreben, unterminierte die konservative Sorge um einen Niveauverlust das Gleichheitsziel, indem es Leistungs- und Qualitätsaspekte von Bildung diskursiv gegen Gleichheit in Stellung brachte. Leistung wurde dabei einer Vorstellung von Gleichheit entgegensetzt, die als einfache Identität („gleichmachen“) konstruiert wurde. Spezifischer noch traten die Bedenken der konservativen KritikerInnen gegen einen Leistungsabfall in den Sprachen dem Leistungsverständnis der Regierung entgegen, die durch eine Erhöhung der sozialen Gleichheit eine Leistungssteigerung allerdings im Sinne einer höheren Qualifizierung der Gesamtbevölkerung erhoffte.

In Luxemburg konnte aufgrund der kurzen Regierungszeit der GesamtschulbefürworterInnen die Reform nicht in der Organisationspraxis verankert werden, weil sich infolge des WählerInnenvotums und neuer Koalitionsbildungen die sozialdemokratisch-dominierte Regierung in Luxemburg nur auf eine Amtsperiode beschränkte. Mit dem Regierungswechsel 1979 blieb die Gesamtschule - „trotz guter Erfahrungen damit" - ein Schulversuch an einer einzelnen Versuchsschule in Düdelingen.

Die Gesamtschulreform wurde in Luxemburg seither nicht mehr aufgegriffen, selbst nicht von der erneut seit 2013 regierenden, nun liberal-sozialdemokratisch-grünen Koalition. Das sogenannte "Gespenst der Gesamtschule“ sei „im Grunde immer noch ein Tabu“, wie ein/e ExpertIn zusammenfasst (LehrerIn, LinkspolitikerIn, Z. 354). Als ein Grund dafür kann die Heftigkeit der damaligen öffentlichen Debatte gesehen werden, die insbesondere von der konservativen Tageszeitung Luxemburger Wort forciert wurde:

Die Gesamtschule war hier in Luxemburg für viele ein Schreckgespenst, das wurde dann natürlich polemisch diskutiert und nicht sachlich behandelt, denn es wurde zum $\mathrm{Ob}$ jekt einer parteipolitischen polemischen Auseinandersetzung zwischen Konservativen und etwas fortschrittlicheren Leuten. (LehrerIn, leitende Funktion in sozialdemokratischer Partei, Z. I68-I7I)

Darüber hinaus ortet ein/e InterviewpartnerIn in Luxemburg jedoch auch eine „Angst vor der Heterogenität, die versteckte Angst vor der Heterogenität der Klasse“ (LehrerIn 2, Gewerkschaft, Z. 949-950), wobei Luxemburg immer schon von einer hohen Diversität in den Schulklassen geprägt gewesen sei. Nachdem sich diese seit der Reform bis heute zumindest bezogen auf die Diversität der nationalen Herkunft der Schulkinder noch 
weiter erhöht hat, kann darin ein weiterer Grund gesehen werden, warum die Gesamtschule mit ihren, in der Öffentlichkeit wenig wahrgenommenen internen Differenzierungsmodellen immer weniger als adäquates Reformprojekt angesichts der Heterogenität an Luxemburgs Schulen erschien :

(D)as Problem ist, dass die Leistung, das Leistungsniveau der Schüler viel unterschiedlicher ist als damals, durch das Sprachenproblem. (...) Das ist das Hauptproblem, also ich seh das so, also eine Gesamtschule jetzt zu machen, wenn man nicht vorher was ändert, das wird eigentlich nicht ... also ich kann mir nicht vorstellen, dass Schüler mit so großen Unterschieden, wie sie bei uns in den verschiedenen Sprachen existieren, dass die in einer Klasse zusammen lernen könnten. Das ist fast nicht möglich, man muss ja auch den Standpunkt des Lehrers sehen, der muss ja auch nen Unterricht organisieren. Und wenn sich in den ersten Schuljahren so große Unterschiede sich entwickeln, und dann ist es irrsinnig zu verlangen, plötzlich in der 7. Klasse eine Gesamtschule zu haben. Wir haben ja ein enseignement fondamental, also Sie kennen ja unser System, wo dann in den ersten Jahren sehr große Probleme entstehen, hauptsächlich für die ausländischen Kinder, die einen portugiesischen Hintergrund haben, weil die einfach das Deutsche nicht verdauen. Die werden dann, ja eliminiert in diesem Bereich, und weil ja auch sehr viel auf Deutsch läuft, verstehen sie auch eine ganze Reihe von anderen Elementen nicht im Unterricht. (LehrerIn I, Gewerkschaft, Z. 62-63, 87-99)

Leistungsprobleme werden im multilingualen und -kulturellen Kontext von Luxemburg somit vor allem als Sprachprobleme konstruiert. In Luxemburg wird die Gesamtschule mit ihrem Gleichheitsfokus als bildungspolitisch nicht durchsetzbar gegen die nationale Spezifik der Mehrsprachigkeit gesehen, weil bestimmte sprachliche Leistungen von politischen AkteurInnen als prioritär gesetzt werden und die Gesamtschule diese nicht zu sichern verspricht.

\section{Berufsständische Konfliktlinien}

Gegenüber dem, medial verstärkten Gleichheits- und Leistungsdiskurs, der die Debatte um die Gesamtschule auch in Luxemburg trug, weisen eine genaue Quellenlektüre und die ExpertInnengespräche jedoch auch auf weitere, weniger beachtete Interessenskonflikte hin, die eine nicht unwichtige Rolle beim Scheitern der Reform spielten. Die Gesamtschule sollte nämlich nicht nur eine gemeinsame Schule für alle Kinder sein; sie impliziert auch eine gemeinsame Schule für alle LehrerInnen. Dies war jedoch keineswegs im berufspolitischen Interesse aller LehrerInnengruppen, vor allem nicht der SekundarlehrerInnen, die als "GymnasialprofessorInnen“ weit höhere Gehälter als andere LehrerInnen bezogen. Diese mit einer Gesamtschulreform verbundenen Sta- tus- und Gehaltskonflikte unter LehrerInnen wurden in Luxemburg nicht offen ausgetragen, wie ein/e befragte/r ExpertIn schildert:

(D)iese allgemeine oder weitgehende Ablehnung der Gesamtschule hat noch einen anderen Hintergrund: Das hieß dann wohl auch, Lehrer sind Lehrer, ob sie nun in der Grundschule unterrichten oder an der Mittelstufe oder am Gymnasium (...). Daher kamen von den Lehrervereinigungen die Bestrebungen, Gesamtschule, dann werden wir auch gleich besoldet wie die anderen, oder ähnlich, oder mindestens in unserem jetzigen Gehalt höhergestellt, und das wollten natürlich die sogenannten Professoren der höheren Schulen dann nicht. Und das war auch eine gewerkschaftliche Sache. (...)

Das hat man natürlich nicht nach außen hin so betont, aber das stand im Hintergrund immer: Wenn wir das mitmachen, dann kommen die Lehrer und sagen, dann, bitte schön, wir sind ja auch anerkannt jetzt, wir wollen eine tranche supplémentaire, wie das so geheißen hat, in unserem Gehalt. Das war in den allermeisten Köpfen der Sekundarlehrer drin, den Lehrern gegenüber. (LehrerIn, HistorikerIn, Leitung Programmkommission, Z. 185-196, 248-252)

Diese offenbar eher hintergründig geführten Interessenskonflikte weisen darauf hin, dass trotz des vermeintlich von vielen geteilten Prinzips der Chancengleichheit durchaus nicht alle AkteurInnen dasselbe Interesse an seiner tatsächlichen Realisierung hatten, da die GrundschullehrerInnen „als ein trojanisches Pferd in den Sekundarschulen" abgelehnt wurden (LehrerIn, LinkspolitikerIn, Z. 325-329). Von dem linken LehrerInnenverein wurde dieses Motiv des Erhalts von Privilegien bereits Ende der 1970er Jahre kritisiert. Die "hysterische Antigesamtschulkampagne“ des Vereins der SekundarschullehrerInnen erkläre „sich zu einem großen Teil aus der Verquickung ihrer Interessen mit der Existenz eines auf Ungleichheit aufgebauten Schulsystems; mit der Existenz eines privilegierten Schultyps, der eigene Privilegien garantiert (größeres soziales Prestige, bessere Bezahlung, bessere Arbeitsbedingungen)“ (Bulletin CLEC 1979, I).

Vor diesem Hintergrund wird deutlich, dass es bei der Legitimation der Gesamtschule nie nur um Fragen der Un-/Gleichheit von SchülerInnen allein geht, sondern diese Ungleichheiten eine politische Lösung der Frage der Un-/Gleichheit von LehrerInnen erfordern würden (Rothmüller 2015). Häufig wird diese jedoch, so auch in Luxemburg, nicht explizit zum Gegenstand bildungspolitischer Aushandlungsprozesse gemacht, und meist auch gar nicht als Problem gesehen, das mit Bildungsgleichheiten verbunden ist.

Ein weiterer Grund, warum die Gesamtschule in $\mathrm{Lu}-$ xemburg schließlich an "massiven Widerständen der Lehrer" scheiterte (LehrerIn, leitende Funktion in sozialdemokratischer Partei, Z. 52), kann darin gesehen 
werden, dass in Luxemburg SekundarlehrerInnen traditionell einen sehr hohen Status im Vergleich zu anderen Ländern hatten. Luxemburg besaß bis 2003 keine eigene Universität, weshalb GymnasialprofessorInnen auch bildungspolitisch einflussreich waren, weil sie als nationale intellektuelle Elite für Parteipolitik und Gewerkschaften rekrutiert wurden. ${ }^{5}$

In diesem Kontext wird relevant, dass die im Zuge der Gesamtschule befürchteten Niveauverluste in den Sprachkenntnissen mit der Reproduktionsweise der nationalen Elite eng verwoben waren:

Also wenn man die Geschichte der Erziehung in Luxemburg sieht, (...) also war das von Anfang an immer erstens eine große Angst, dass man das Niveau nicht erreichen würde, was man bräuchte, um hernach zur Universität ins Ausland zu gehen. Das war eine große Befürchtung, ist es heute auch noch bei vielen Eltern. Und das zweite, das war, dass man, wenn man etwas öffnen würde und sagen wir, die Kinder etwas mehr fördern würde, dass man hernach zu viele Intellektuelle hätte, die arbeitslos wären (LehrerIn, leitende Funktion sozialdemokratische Partei, Z.I27-I33).

Es ging also explizit bei der Reform auch um soziale Exklusion - von statusniederen LehrerInnen wie von niedrigen sozialen Schichten. In Luxemburg konnten schließlich die SekundarlehrerInnen 1980 zur Gesamtschulinitiative resümieren: „Es hatte ein Klimawechsel sein sollen, es wurde ein kurzes, heftiges Gewitter daraus." (Bulletin spécial APESS I980, 68)

\section{Schlussfolgerungen}

Zusammenfassend kann am Beispiel Luxemburgs gezeigt werden, dass im Gesamtschuldiskurs Leistung und Gleichheit je nach bildungspolitischer Position sehr unterschiedlich verknüpft werden: Sozialdemokratische ReformbefürworterInnen verfolgen mit der Strukturreform das Ziel von Gleichheit im Sinne eines Ausgleichs sozialer Benachteiligung durch gemeinsamen Unterricht bei nur interner Differenzierung. Leistung wird am Ideal demokratischer BürgerInnenschaft und Hebung des kulturellen Niveaus der Bevölkerung gemessen, und als ein Prozess begriffen, der auch bei Jugendlichen noch nicht abgeschlossen ist. Konservative ReformgegnerInnen hingegen sehen in Gleichheit und Leistung einen Widerspruch. Obwohl sich auch Konservative teils positiv auf das Konzept der Chancengleichheit beziehen, fürchteten GesamtschulgegnerInnen einen allgemeinen Leistungsrückgang vor allem im Bereich der klassischen Bildung und Sprachen durch wiederholte Gleich-

5 Dies zeigte sich auch bei den befragten ExpertInnen, von denen nahezu alle am Beginn ihrer beruflichen Karriere an verschiedenen luxemburgischen Gymnasien unterrichtet hatten. heitsinterventionen. Weil Begabungen früh festgelegt und wenig veränderbar seien, würde eine gemeinsame Schule für alle Kinder zu einem Niveauverlust führen. Gleichheit und Leistung werden so im konservativen Gesamtschuldiskurs verbunden als "Gleichschaltung in der Mittelmäßigkeit“. Auch dieser Sorge widersprechende empirische Befunde und verschiedene Arten einer internen Differenzierung der Kinder in den meisten Gesamtschulmodellen ändern meist wenig an diesen Überzeugungen der KritikerInnen. Eine zu starke interne Differenzierung ist, wie gezeigt, wiederum Gegenstand linker Kritik an einer übermäßigen Orientierung der Gesamtschule an einem Leistungsprinzip, das als Teil kapitalistischer Ausbeutung begriffen wird. Linke bildungspolitische AkteurInnen betonen demgegenüber den egalisierenden Charakter der Gesamtschule und sehen sie als Teil einer notwendig gesamtgesellschaftlichen Veränderung hin $\mathrm{zu}$ sozialer und politischer Gleichheit.

Mit der Gesamtschuldebatte sind somit verschiedene Gleichheitsideale und Leistungsvorstellungen verknüpft, wobei sich das Prinzip der punktuellen Chancengleichheit gegenüber Prinzipien, welche die durchgehende Gewährleistung gleicher Chancen oder Ergebnisgleichheit beinhalten, durchgesetzt hat. Im Prinzip der Startchancengleichheit kommt Leistung als Ungleichheitskriterium eine zentrale Rolle zu. Wie die im ersten Teil des Beitrags berichteten empirischen Befunde zeigen, scheint die Gesamtschule zwar gegenüber gegliederten Schulsystemen im Vorteil zu sein, ein im Vergleich höheres Maß an Chancengleichheit zu gewährleisten, ohne dass Abstriche beim Bildungs-Output - etwa bei Kompetenzen - gemacht werden müssten. Allerdings ist es nicht die Gesamtschule allein, welche hier $\mathrm{zu}$ Gleichheit und (System-)Leistung beiträgt, sondern es ist auch eine Frage ihrer Ausgestaltung im Detail und begleitender Maßnahmen wie etwa der Mittelausstattung zur Bewältigung der Heterogenität (z. B. zusätzliche Unterstützungslehrpersonen). Diese Befunde sind jedoch unterschiedlich anschlussfähig an nationale Reformdebatten. Die (System-)Leistung der Gesamtschule in Luxemburg wurde an ihrem Beitrag zu gesellschaftlicher, sozialer und politischer Gleichheit, aber auch an ihrem Einfluss auf bestimmte Leistungsmerkmale (Mehrsprachigkeit) der SchülerInnen gemessen.

Bis heute gilt das Thema in Luxemburg als Tabu, an dem aufgrund des polarisierend-eskalierenden Debattenverlaufs in den 1970ern und der Problemkonstruktion der ,heterogenen Klasse' niemand ernsthaft zu rühren wagt. Im internationalen Vergleich zeigt sich dabei, dass für die bildungspolitische Reform einer $\mathrm{Ge}-$ samtschule nicht zuletzt ausschlaggebend war, wie lange in den Reformländern jeweils SozialdemokratInnen an der Macht waren. Sowohl in Österreich, wo 1970 und I97I die SPÖ die Wahlen gewann, als auch etwas später 
in Luxemburg wurden Gesamtschulmodelle von sozialdemokratischen Regierungen in Bildungsprogramme aufgenommen (Bucher 2013, 80). Allerdings wurden sie in beiden Ländern trotz sozialdemokratisch geführter Regierungen nur in Ansätzen umgesetzt. Das Engagement der SozialdemokratInnen für die Gesamtschule war in Deutschland ausschlaggebend für die Reformen: Gesamtschulen wurden versuchsweise u.a. in Hessen eingeführt, wo zu diesem Zeitpunkt SozialdemokratInnen an der Regierung beteiligt waren (Oelkers 2006, 58). Busemeyer und Nikolai (2010, 506) betonen dazu, dass die hohe Kontinuität der sozialdemokratische Reformpolitik in Skandinavien die eigentliche Voraussetzung für die dortige Ausweitung der Bildungschancen war. Dass die Gesamtschule in der BRD nicht flächendeckend eingeführt wurde, wird umgekehrt auf den hartnäckigen Widerstand von CDU und CSU zurückgeführt. Die Zahl der Gesamtschulen wuchs in den I980er und I990er Jahren trotzdem - weil die Nachfrage von Eltern hoch war und dieser Schultyp vielfach auf Wunsch von Eltern etabliert wurde (Wenzler 2003, 77).

Neben dieser eher allgemeinen Einsicht in die Bedeutung kontinuierlicher Reformpolitik weisen sowohl die Quellenanalyse als auch Interviews mit bildungspolitischen AkteurInnen in Luxemburg auf standespolitische Kämpfe, nämlich auf den massiven Widerstand einiger Gruppen von SekundarlehrerInnen "im Hintergrund“ der Gesamtschulinitiative hin. Die berufspolitischen Konsequenzen einer Gesamtschule werden bis heute wenig wissenschaftlich wie bildungspolitisch adressiert: Aus Perspektive damaliger wie heutiger bildungspolitischer AkteurInnen impliziert die Gesamtschule nicht notwendigerweise eine Gleichheit unter LehrerInnen. Die Kooperation von LehrerInnen im Rahmen einer gemeinsamen Schule für alle wird aktuell zwar auch in Österreich prinzipiell als notwendige Zusammenarbeit gesehen, aber zugleich eine stärkere ,Leistungsförderung', etwa durch ein „leistungsförderndes Dienstrecht" (Schmid 20I4, 8I) eingefordert. Der Aspekt der Gleichheit steht somit nur bei SchülerInnen, nicht jedoch bezogen auf LehrerInnen im Mittelpunkt der bildungspolitischen Legitimation von Gesamtschulreformen. Die Kontinuität und Konsequenz im Verfolgen bildungspolitischer Reformen durch dauerhafte sozialdemokratische Regierungen war insofern nicht zuletzt notwendig, um die Gesamtschule gegen den konservativen Widerstand der SekundarlehrerInnen, der auch in Skandinavien oder Frankreich zu beobachten war (für Finnland vgl. Ahonen 2002, I76; für Schweden Ratzki 2003, I28; für Frankreich vgl. Werry 2003, 94), durchzusetzen. Vor diesem Hintergrund kann Erfolg oder Scheitern der Gesamtschulreform als kontextspezifisches Ergebnis des Zusammenspiels von Berufspolitik und nationaler Elitereproduktion mit gesellschaftspolitischen Dynamiken verstanden werden.

\section{Literatur}

Ahonen, Sirkka (2002). From an Industrial to a Post-industrial Society: changing conceptions of equality in education, in: Educational Review, Vol. 54(2), I73-I8I.

Allmendinger, Jutta (1989). Educational systems and labor market outcomes, in: European Sociological Review Vol. 5, 23I-250.

Alon, Sigal/Marta Tienda (2007). Diversity, opportunity, and the shifting meritocracy in higher education, in: American Sociological Review, Vol. 72(4), 487-5II.

Bogner, Alexander/Beate Littig/Wolfgang Menz (Hg.) (2005). Das Experteninterview. Theorie, Methode, Anwendung. 2. Aufl., Wiesbaden.

Bruneforth, Michael/Christoph Weber/Johann Bacher (2012). Chancengleichheit und garantiertes Bildungsminimum in Österreich, in: Barbara HerzogPunzenberger (Hg.): Nationaler Bildungsbericht Österreich 2012. Band 2, Graz, I89-229.

Baumert, Jürgen/Petra Stanat/Rainer Watermann (2006). Schulstruktur und die Entstehung differenzieller Lern- und Entwicklungsmilieus, in: Jürgen Baumert/ Petra Stanat/Rainer Watermann (Hg.): Herkunftsbedingte Disparitäten im Bildungswesen: Differenzielle Bildungsprozesse und Probleme der Verteilungsgerechtigkeit, Wiesbaden, 95-I88.

Benz, Arthur (2009): Politik in Mehrebenensystemen, Wiesbaden.

Boudon, Raymond (1974). Education, Opportunity, and Social Inequality, New York.

Bönsch, Manfred (2006). Gesamtschule. Die Schule der Zukunft mit historischem Hintergrund, Baltmannsweiler.

Bourdieu, Pierre/Jean-Claude Passeron (197I). Die Illusion der Chancengleichheit. Untersuchungen zur Soziologie des Bildungswesens am Beispiel Frankreichs, Stuttgart.

Bourdieu, Pierre/Luc Boltanski/Monique de Saint Martin/ Pascale Maldidier-Pargamin (198I). Titel und Stelle. Über die Reproduktion sozialer Macht, Frankfurt am Main.

Bourdieu, Pierre (1997). Das Elend der Welt. Zeugnisse und Diagnosen alltäglichen Leidens an der Gesellschaft, Konstanz.

Bucher, Anton A. (2013). Armut und Bildungschancen: Gesamtschule als Ausweg? Mit einem Fokus auf Österreich, in: Helmut P. Gaisbauer/Elisabeth Kapferer/Andreas Koch/Clemens Sedmak (Hg.): Armut und Wissen. Reproduktion und Linderung von Armut in Schule und Wissenschaft, Wiesbaden, 69-88.

Bulletin IR (Bulletin de l'Association des instituteurs réunis du Grand-Duché de Luxembourg) (1973). Einführung in den Fragenkreis der Integrierten Gesamtschule (I. Teil), Bulletin 24(3), 3-40. 
Bulletin APESS (Association des professeurs de l'enseignement secondaire et supérieur) (1978). Informations- und Diskussionsabend im Lycée Michel Rodange. Einseitige Stimmungsmache gegen den Tronc Commun, II.

Bulletin d'information CLEC (Cercle de liaison des enseignants critiques) (1979). Numéro special. Tronc commun: Ein luxemburgisches Gesamtschulmodell?

Bulletin spécial APESS (Association des professeurs de l'enseignement secondaire et supérieur du GrandDuché de Luxembourg) (1980). Schule und Politik. Beitrag von Henri Koch, 67-70.

Chambers, Clare (2009). Each outcome is another opportunity: Problems with the Moment of Equal Opportunity, in: Politics, Philosophy and Economics, Vol. 8(4), 374-400.

Coleman, James S./Ernest Q. Campbell/Carol J. Hobson/ James McPartland/Alexander M. Mood/Frederic D. Weinfeld/Robert L. York (1966). Equality of Educational Opportunity. Washington, DC: U.S. Department of Health, Education and Welfare.

Courrier de l'éducation nationale (1977). Le Tronc Commun à Luxembourg. Une réforme fondamentale. Nr. A4, hg. v. Ministère de l'Éducation Nationale, Juli 1977.

Crul, Maurice/Philipp Schnell/Barbara Herzog-Punzenberger/Maren Wilmes/Marieke Slootman/Rosa AparicioGomez (2012). School careers of second-generation youth in Europe: Which education systems provide the best chances for success? In: Maurice Crul/Jens Schneider/Frans Lelie (Hg.): The European Second Generation Compared. Does the Integration Context Matter? Amsterdam, 99-I77.

Deutscher Bildungsrat (1969). Einrichtung von Schulversuchen mit Gesamtschulen, Stuttgart.

Dworkin, Ronald (2000). Sovereign virtue, Cambridge, MA.

d'Letzeburger Land (1977a). Gerechtigkeit aus der Retorte. $\mathrm{Zu}$ den inhaltlichen Problemen der Gesamtschuldebatte, in: d'Letzeburger Land. Unabhängige Wochenschrift for Politik, Wirtschaft und Kultur, I5.7.1977, 8-9.

d'Letzeburger Land (1977b). Chancengleichheit: Utopie und Notwendigkeit. Anmerkungen zu einer Bildungsreform, in: d'Letzeburger Land. Unabhängige Wochenschrift for Politik, Wirtschaft und Kultur, II.II.I977, 5.

École et vie (1976). Versuchsweise oder verallgemeinerte Einführung der Gesamtschule?, in: École et vie. Revue pédagogique et culturelle trimestrielle, éditée par l'association des instituteurs réunis luxembourg, 8-II.

Hadjar, Andreas/Rolf Becker (Hg.) (2009). Expected and Unexpected Consequences of the Educational Expansion in Europe and the US. Theoretical approaches and empirical findings in comparative perspective, Bern/Stuttgart/Wien.
Hadjar, Andreas/Joël Berger (20II). Geschlechtsspezifische Bildungsungleichheiten in Europa, in: Andreas Hadjar (Hg.): Geschlechtsspezifische Bildungsungleichheiten, Wiesbaden, 23-54.

Hadjar, Andreas/Joël Berger (20I0). Dauerhafte Bildungsungleichheiten in Westdeutschland, Ostdeutschland und der Schweiz: Eine Kohortenbetrachtung der Ungleichheitsdimensionen soziale Herkunft und Geschlecht, in: Zeitschrift für Soziologie, Vol. 39(3), I82-2OI.

Hadjar, Andreas/Christiane Gross (Hg.) (20I6). Education Systems and Inequalities, Bristol.

Hadjar, Andreas/Antoine Fischbach/Romain Martin/Susanne Backes (2015). Bildungsungleichheiten im luxemburgischen Bildungssystem, in: Ministère de l'Éducation nationale, de l'Enfance et de la Jeunesse, SCRIPT und Université du Luxembourg, FLSHASE (Hg.): Bildungsbericht Luxemburg 2015. Analysen und Befunde, Luxembourg, 34-56.

Herrlitz, Hans-Georg (20I3). Der Streit um die Gesamtschule in Niedersachsen. Ein dokumentarischer Rückblick auf die Landtagsdebatten I967 - 2012. Göttinger Beiträge zur erziehungswissenschaftlichen Forschung 34, Institut für Erziehungswissenschaft, Georg August-Universität Göttingen.

Herrlitz, Hans-Georg/Dieter Weiland/Klaus Winkel (Hg.) (2003). Die Gesamtschule. Geschichte, internationale Vergleiche, pädagogische Konzepte und politische Perspektiven, Weinheim/München.

Keim, Wolfgang (2004). Gesamtschule als Antwort auf die Globalisierung? Die aktuelle Schulsystemdebatte im Spiegel historischer und internationaler Erfahrungen, in: Gerd Steffens/Edgar Weiß (Hg.): Globalisierung und Pädagogik. Frankfurt am Main, 27I-293.

Koall, Iris/Verena Bruchhaben/Friederike Höher (Hg.) (2007). Diversity outlooks: Managing Diversity zwischen Ethik, Profit und Antidiskriminierung, Münster.

MENFP/EMACS (2010). PISA 2009: Nationaler Bericht Luxemburg, Luxembourg.

Meulemann, Heiner (1992). Gleichheit, Leistung und der Wandel oder Nicht-Wandel von Werten - Warum die Wahrnehmung realisierter Gleichheit in der Bundesrepublik Deutschland sich nicht verändert hat, in: Helmut Klages/Hans-Jürgen Hippler/Willi Herbert (Hg.): Werte und Wandel. Ergebnisse und Methoden einer Forschungstradition, Frankfurt am Main/New York, IOO-I26.

Miller, David (2002). Liberalism, Equal Opportunities and Cultural Commitments, in: Paul Kelly (Hg.): Multiculturalism Reconsidered: Culture and Equality and its Critics, Malden, 45-6I.

MENEJ (Ministère de l'Éducation nationale, de l'Enfance et de la Jeunesse, SCRIPT) \& Université du Luxem- 
bourg, FLSHASE (Hg.) (2015). Bildungsbericht Luxemburg 2015. Analysen und Befunde. Luxembourg.

Müller, Walter/Wolfgang Karle (1993). Social Selection in Educational Systems in Europe, in: European Sociological Review, Vol. 9, I-23.

Müller, Walter/Susanne Steinmann/Reinhart Schneider (1997). Bildung in Europa, in: Stefan Hradil/Stefan Immerfall (Hg.): Die westeuropäischen Gesellschaften im Vergleich, Opladen, I77-246.

Nullmeier, Frank (200I). Politikwissenschaft auf dem Weg zur Diskursanalyse? In: Reiner Keller/Andreas Hirseland/Werner Schneider/Willy Viehöver (Hg.): Handbuch Sozialwissenschaftliche Diskursanalyse, Opladen, 285-312.

Oelkers, Jürgen (2006). Gesamtschule in Deutschland. Eine historische Analyse und ein Ausweg aus dem Dilemma, Weinheim/Basel.

OECD Organisation for Economic Cooperation and Development (2005). School Factors Related to Quality and Equity. Results from PISA 2000, Paris.

OECD Organisation for Economic Cooperation and Development (20II). PISA 2009 Ergebnisse: Was macht eine Schule erfolgreich? Lernumfeld und schulische Organisation in PISA (Band IV), Paris.

Protokoll der 58. Parlamentssitzung vom 13.03.1979. V. Projet de loi portant création d'un premier cycle intégré de l'enseignement postprimaire (tronc commun) - Nr. 2235 - Rapport de la Commission de l'Education nationale, Luxembourg, 3838-3854.

Protokoll der 60. Parlamentssitzung vom 15.03.1979. II. Projet de loi portant création d'un premier cycle intégré de l'enseignement postprimaire (tronc commun) Nr. 2235 - Discussion générale, Luxembourg, 39224038.

Pfeffer, Fabian (2008). Persistent inequality in educational attainment and its institutional context, in: European Sociological Review, Vol. 24, 543-565.

Ratzki, Anne (2003). Schweden. Individuell lernen in der gemeinsamen Schule, in: Hans-Georg Herrlitz/ Dieter Weiland/Klaus Winkel (Hg.): Die Gesamtschule. Geschichte, internationale Vergleiche, pädagogische Konzepte und politische Perspektiven, Weinheim/ München, III-I32.

Rawls, John, I975 [197I]. Eine Theorie der Gerechtigkeit, Frankfurt am Main.

Rothmüller, Barbara (2OI4). Bildungspolitische Theorieeffekte und ihre Komplizenschaft mit Ungleichheiten, in: Jens Kastner/Ruth Sonderegger (Hg.): Pierre Bourdieu und Jacques Rancière. Emanzipatorische Praxis denken, Wien, I47-I8I.

Rothmüller, Barbara (2015). Die Legitimation einer Gesamtschule: Historische Bruchlinien und Randzonen von Gleichheit in der Debatte um das „Paradepferd sozialistischer Bildungspolitik“, in: Maria Dammayr/ Doris Graß/Barbara Rothmüller (Hg.): Legitimität.
Gesellschaftliche, politische und wissenschaftliche Bruchlinien der Rechtfertigung, Bielefeld, 199-225.

Schmid, Kurt (20I4). Erfolgsfaktoren für eine "gemeinsame Schule". Strukturvergleiche und Analysen anhand ausgewählter Länder, Wien, ibw-Forschungsbericht Nr. 178.

Schnepf, Sylke Viola (2002). A Sorting Hat That Fails? The transition From Primary To Secondary School in Germany. Innocenti Working Papers No. 92, UNICEF Innocenti Research Centre, Florence.

Schubert, Frank/Rolf Becker (2010). Social Inequality of Reading Literacy. A longitudinal analysis with crosssectional data of PIRLS 200 I and PISA 2000 utilizing the pairwise matching procedure, in: Research in Social Stratification and Mobility (Special issue: New Developments in Education Transitions Research), Vol. 28 (I), I09-I33.

Stelzer-Orthofer, Christine/Bacher, Johann (2008). Schulsysteme, Wohlfahrtsstaatswelten und schulische Integration von Kindern mit Migrationshintergrund, in: Bettina Leibetseder/Josef Weidenholzer (Hg.): Integration ist gestaltbar, 65-93.

Swift, Adam (2004). Would perfect mobility be perfect?, in: European Sociological Review, Vol. 2O(I), I-II.

Van de Werfhorst, Herman G./Jonathan B. Mijs (2010). Achievement Inequality and the Institutional Structure of Educational Systems, in: Annual Review of Sociology, Vol. 36, 407-428.

Vester, Michael (2006). Die ständische Kanalisierung der Bildungschancen. Bildung und soziale Ungleichheit zwischen Boudon und Bourdieu, in: Werner Georg (Hg.): Soziale Ungleichheit im Bildungssystem. Eine empirisch-theoretische Bestandsaufnahme, Kons$\operatorname{tanz}$, 13-54.

Von Below, Susanne (2009). Bildungssysteme im historischen und internationalen Vergleich, in: Rolf Becker (Hg.): Lehrbuch der Bildungssoziologie, Wiesbaden, I3I-I53.

Wenzler, Ingrid (2003). Bundesrepublik Deutschland. Die Gesamtschule: Kräfte und Gegenkräfte im bildungspolitischen Konflikt, in: Hans-Georg Herrlitz/Dieter Weiland/Klaus Winkel (Hg.) (2003): Die Gesamtschule. Geschichte, internationale Vergleiche, pädagogische Konzepte und politische Perspektiven. Weinheim/ München, 65-86.

Werry, Hanno (2003). Frankreich. Von der republikanischen Tradition zur demokratischen Praxis, in: Hans-Georg Herrlitz/Dieter Weiland/Klaus Winkel (Hg.) (2003): Die Gesamtschule. Geschichte, internationale Vergleiche, pädagogische Konzepte und politische Perspektiven, Weinheim/München, 87-IIO.

Wocken, Hans (1988). Leistung und Integration, in: Hans Wocken/Georg Antor/Andreas Hinz (Hg.): Integrationsklassen in Hamburger Grundschulen - Bilanz eines Schulversuchs. Hamburg, 379-396. 
Young, Michael (1958). The Rise of the Meritocracy, London.

\section{AutorInnen}

Andreas Hadjar, 1974, Professor für Bildungssoziologie, Institute of Education and Society, Université du Luxembourg. Forschungsinteressen: Bildungssoziologie, Sozialstrukturanalyse, Politische Soziologie, Internationale Vergleiche, Methoden der empirischen Sozialforschung. Aktuelle Publikationen: „Education Systems and Inequalities" (gem. mit Christiane Gross, Policy Press), „Die politische Dimension der Bildung. Zu Jutta Allmendinger: ,Bildungsarmut: Zur Verschränkung von Bildungs- und Sozialpolitik'“ (gem. mit Christiane Gross, Soziale Welt, Sonderband 2I), "Does upward social mobility increase life satisfaction? A longitudinal analysis using British and Swiss panel data" (gem. mit Robin Samuel, Research in Social Stratification and Mobility 39).

Barbara Rothmüller, 1982, Projektmitarbeiterin, Institute of Education and Society, Université du Luxembourg/ Graduate School of Education, Stanford University, US. Forschungsinteressen: Bildungssoziologie, -theorie und -geschichte, soziale Ungleichheiten, Nationalismus und StaatsbürgerInnenschaft, politische Philosophie, Diskurs- und Praxistheorien. Aktuelle Publikationen: „Educating future citizens in between Mischkultur nationalism and authorities: traces from teachers' journals “ (gem. mit Matias Gardin und Ragnhild Barbu, History of Education 44/5). Legitimität. Gesellschaftliche, politische und wissenschaftliche Bruchlinien der Rechtfertigung (hg. gem. mit Maria Dammayr und Doris Graß, transcript 2015). 